\title{
No Difference in Clinical Outcome between Middle Turbinate Resection vs Inferior Turbinoplasty in Patients with Rhinitis
}

\author{
Danny Kit Chung Wong ${ }^{1}$, Lokman Saim² ${ }^{2}$ Aminuddin Saim ${ }^{3}$
}

\begin{abstract} outcome test-22 (SNOT-22), and visual analog scale (VAS) in patients with rhinitis. pre- and postoperatively via PNIF, SNOT-22, VAS, and endoscopic examination. group complained of intermittent long-term epistaxis. morbidity of postoperative bleeding.

Keywords: Allergic rhinitis, Cohort study, Endoscopy, Quality of life, Rhinomanometry, Surgery, Turbinates.

Otorhinolaryngology Clinics: An International Journal (2019): 10.5005/jp-journals-10003-1324
\end{abstract}

Introduction:To date, there is no optimal surgical management for rhinitis. Current techniques include inferior turbinate resection or turbinoplasty (ITP), septoplasty, and submucosal resection. Middle turbinate resection (MTR) at present is used as part of endoscopic sinus surgery to prevent adhesions and recurrent disease. In this study, the outcomes of MTR and ITP were compared via peak nasal inspiratory flow (PNIF), sinonasal

Materials and methods: In a prospective study, 22 consented patients with rhinitis from the otolaryngology head and neck surgery outpatient clinic were selected and underwent surgery. Twelve patients had MTR and 10 patients had ITP with a 6-month follow-up. Patients were evaluated

Results: Both treatment groups showed statistically significant improvements $(p<0.01)$ in PNIF, SNOT-22, and VAS scores postoperatively in early and late follow-up when compared preoperatively. There was a median increase of 57-58\% in PNIF, a decrease in SNOT-22 scores by 53-80\%, and a decrease in 64-78\% VAS scores at the 6-month follow-up. There were no significant differences between scores when comparing the MTR and ITP groups. ITP group had more early postoperative bleeding compared to the MTR group $(p<0.05)$ and a few patients from the ITP

Conclusion: Middle turbinate resection was observed to be as effective as ITP to reduce the signs and symptoms of rhinitis and has a lower

\section{INTRODUCTION}

Rhinitis is a worldwide problem. It can be divided into nonallergic rhinitis (NAR) and allergic rhinitis (AR) based on whether it is an lgE or non-lgE mediated inflammation of the nasal mucosa. ${ }^{1}$ Nonetheless, these patients experience similar symptoms of nasal dysfunction that include symptoms of itchy eyes, nose or palate, sneezing, rhinorrhea, and nasal obstruction. It has been postulated that these symptoms are due to a dysfunction of the autonomic system and sensory nerve imbalance in the nasal mucosa causing vasodilation and glandular secretion of the mucosa. ${ }^{2}$

The optimal management for rhinitis is not well established. The current recommended medical treatments include intranasal steroids, oral antihistamines, and immunotherapy for patients with atopy. ${ }^{3}$ Unfortunately, these medications have been reported to be ineffective in up to $37 \%$ of patients. ${ }^{4}$ In patients who have failed medical management, the surgical treatments are limited to the reduction of inferior turbinate (IT) volumes via turbinectomy, submucosal diathermy, or turbinoplasty. Although many studies have reported good outcome in terms of nasal congestion for IT volume reduction surgery, surgical treatment continues to be largely surgeon dependent and has been associated with complications, such as, atrophic rhinitis, thermal tissue damage, and primary hemorrhage. ${ }^{5,6}$

Middle turbinate resection (MTR) has been documented in the literature since 1921 and described as part of the procedure during a complete ethmoid clearance. ${ }^{7}$ At present, surgeons who favor MTR perform it in endoscopic sinus surgery for the purposes of decreasing synechiae formation, improving the sinus outflow tract, and for better endoscopic visualization postoperatively. ${ }^{8}$

\footnotetext{
1,2 Department of Otorhinolaryngology-Head and Neck Surgery, KPJ Healthcare University College, Nilai, Negeri Sembilan, Malaysia

${ }^{3}$ Department of Otorhinolaryngology-Head and Neck Surgery, KPJ Ampang Puteri Specialist Hospital, Ampang, Selangor, Malaysia

Corresponding Author: Danny Kit Chung Wong, Department of Otorhinolaryngology-Head and Neck Surgery, KPJ Healthcare University College, Nilai, Negeri Sembilan, Malaysia, Phone: +60123878279, e-mail: drdannykcwong@gmail.com.
}

How to cite this article: Wong DKC, Saim L, Saim A. No Difference in Clinical Outcome between Middle Turbinate Resection vs Inferior Turbinoplasty in Patients with Rhinitis. Int J Otorhinolaryngol Clin 2019;11(1):1-4.

Source of support: This study was supported by a grant by the Malaysian Society of Otorhinolaryngologists Head \& Neck Surgeons (MSOHNS)

Conflict of interest: None

The MT physiology is thought to be functionally similar to the IT. However, due to its size, it plays a smaller role in nasal airconditioning or sensing of nasal airflow. This means that a MTR with preservation of the IT could possibly allow the nose to maintain better physiological nasal airflow thereby reducing the risk of atrophic rhinitis. ${ }^{9}$

The mucosa of the MT contains numerous vasodilator and trigeminal nerve afferents at the mucous membrane. It has also been shown to be a viable source of epithelium due to its impressive regenerative qualities. ${ }^{10,11}$ Hence, we believed that by removing the MT, the bulk of the autonomic supply to the nasal cavity would

() The Author(s). 2019 Open Access This article is distributed under the terms of the Creative Commons Attribution 4.0 International License (https://creativecommons. org/licenses/by-nc/4.0/), which permits unrestricted use, distribution, and non-commercial reproduction in any medium, provided you give appropriate credit to the original author(s) and the source, provide a link to the Creative Commons license, and indicate if changes were made. The Creative Commons Public Domain Dedication waiver (http://creativecommons.org/publicdomain/zero/1.0/) applies to the data made available in this article, unless otherwise stated. 
be disrupted thereby subsequently reducing the sensory nerverelated symptoms of rhinitis. We believed that at the very least, this procedure would be equivalent to inferior turbinate resection or turbinoplasty (ITP).

\section{Materials and Methods}

\section{Study Design and Ethics Approval}

This study was prospectively conducted on patients at KPJ Ampang Puteri Specialist Hospital, Selangor, Malaysia, between 1 April, 2016 and 30 September, 2016. Our objective was to evaluate the outcome between MTR and ITP groups. Before commencement of the study, full ethical approval was obtained from the Research and Innovation Center of KPJ Healthcare University College (KPJUC/ RIC/PIN/2016/006).

\section{Population}

A total of 22 patients from the otolaryngology head and neck surgery outpatient clinic, KPJ Ampang Puteri Specialist Hospital who met the inclusion criteria were selected to receive a surgical intervention. Inclusion criteria were patients with moderate to severe rhinitis for $\geq 6$ months; bilateral turbinate hypertrophy on endoscopic examination despite a trial of a minimum of 4 weeks of intranasal steroids and oral antihistamines; a SNOT-22 >7, and a PNIF of $<115 \mathrm{~L} /$ minute $( \pm 36 \mathrm{~L} /$ minute). Exclusion criteria were evidence of nasal polyposis, severe septal deviation, previous nasal surgery, or asthma. These patients were consented and then randomized into two surgical treatment groups through an alternating sequence. One group underwent MTR $(n=12)$ and the other ITP $(n=10)$. All patients had a preoperative computed tomography (CT) scan to ensure that they only had very mild inflammatory mucosal sinus disease with disease limited to only the maxillary or anterior ethmoid sinuses not exceeding grade I of the Lund-Mackay Score. ${ }^{12}$

\section{Techniques}

\section{Peak Nasal Inspiratory Flow}

Peak nasal inspiratory flow (PNIF) was measured with a PNIF meter (Clement Clarke International, London, U.K.). ${ }^{13}$ Patients are were asked to hold the PNIF meter horizontally, ensuring that a tight seal was formed around the facemask without constricting the nose and inhaled forcibly through the nose while keeping the mouth close. The greatest measurement was documented. At a value of $<115 \mathrm{~L} /$ minute ( $\pm 36 \mathrm{~L} /$ minute), PNIF correlates well with signs of rhinitis. ${ }^{14}$ PNIF has been shown to be a validated tool for objective assessment of nasal patency and airflow. It has been successfully used for the evaluation of treatment in rhinitis and is one of the most frequently used instruments apart from rhinomanometry and acoustic rhinometry. ${ }^{15}$

\section{MTR}

The superior attachment was cut along its laminar portion with a pair of curved scissors for about $2 / 3$ its length. The scissors was then inverted to cut off the vertical portion of the MT. Hemostasis was established if necessary by temporarily packing the nose with half-inch ribbon gauze soaked in 1:1000 adrenaline. ${ }^{16,17}$

\section{ITP}

A small incision was placed at the head of the IT and a powered intramucosal turbinate blade of $2.9 \mathrm{~mm}$ (Medtronic, Minneapolis, MN) would be introduced submucosally to debulk the tissue and create a pocket in the IT. The blade was then reintroduced again to make a linear incision along the length of the IT, a subperiosteal medial mucosal flap would be raised, and turbinate bone would be removed entirely. The medial flap was then rotated laterally onto itself and surgical dressing was inserted to support the flap. ${ }^{5}$

\section{Postoperative Management on Discharge}

Postoperatively, nasal irrigations with saline solution were prescribed to all patients along with an antihistamine continuously for 6 weeks.

\section{Postoperative Management and Evaluation \\ Descriptive Features and Classification of Rhinitis}

Descriptive features of all patients, such as, age, ethnicity, gender, height, and weight were recorded. Classification of rhinitis was according to ARIA guidelines. Intermittent rhinitis was defined as a nasal symptoms lasting $<4$ days/week or $<4$ weeks/year and persistent rhinitis was defined as nasal symptoms lasting $\geq 4$ days/ week or $\geq 4$ weeks/year. Degree of severity was based on the VAS score, where $>5 / 10 \mathrm{~cm}$ was classified as moderate to severe. ${ }^{18,19}$

\section{Evaluation of Patients}

All patients were evaluated pre- and postoperatively with a PNIF measurement for objective scoring of their nasal obstruction. During each clinic visit, patients underwent an endoscopic evaluation of the nasal cavity and were required to complete a SNOT-22 questionnaire and VAS for subjective assessments of their symptoms and quality of life (QoL). The SNOT-22 comprised of 22 questions encompassing rhinological symptoms (q1-8), ear and facial symptoms (q9-12), sleep function (q13-15), and psychological function (q16-22) on a 5-point scale $(0=$ no problem, 1 = very mild problem, 2 = mild or slight problem, $3=$ moderate problem, $4=$ severe problem, and $5=$ problem as bad as it can be). ${ }^{20}$ A SNOT-22 score of $>7$ may indicate an abnormality. ${ }^{21}$ The VAS is visual analog scale score ranging from 0 ("nasal symptoms not at all bothersome") to $10 \mathrm{~cm}$ ("nasal symptoms, extremely bothersome"), where six parameters were assessed (sneezing, runny nose, postnasal drip, congestion, itchy nose, and total symptoms score).$^{19}$ These tools have been shown to be validated, simple, and reliable with a high positive and negative predictive value. ${ }^{20,22,23}$ Inferior turbinate hypertrophy was documented using a three-point ordinal scale based on the ability to visualize the nasopharynx without decongestion, the posterior aspect of the middle turbinate, middle portion of the turbinate, and only the anterior head $(0=$ no obstruction, $1=$ mild obstruction, 2 = moderate obstruction, or $3=$ severe obstruction). ${ }^{5}$ The measurements of PNIF, SNOT-22, and VAS from the surgical group would be compared against the preoperative scores to determine the effectiveness of treatment.

\section{Immediate Postoperative Evaluation}

Postoperatively, patients were observed on the ward for signs of bleeding and level of pain. The number of nasal bolster changes was recorded to estimate the postoperative blood loss.

\section{Follow-up Evaluation at 2 Weeks, 2 Months, and 6 Months}

Patients were specifically monitored for the following postoperative complications that included bleeding, atrophic rhinitis, adhesions, anosmia, and failure requiring revision procedure.

\section{Statistical Analysis}

Statistical analysis was performed using SPSS V 23 (IBM, Armonk, NY). Wilcoxan signed-rank test with matched pairs was used to evaluate the pre- and postoperative symptoms scores (i.e., PNIF, 
SNOT-22, and VAS). For categorical variables, differences between groups were calculated with Fisher's exact test.

\section{Results}

\section{Comparison of PNIF, SNOT-22, VAS, and Inferior Turbinate Size between MTR and ITP Groups}

A total of 22 patients ( 13 males) aged 17-40 years were treated surgically and followed up for 6 months. All patients completed a PNIF measurement, SNOT-22, and VAS and were examined endoscopically at each clinic visit. There were no significant differences $(p>0.05)$ in demographics or preoperative characteristics for both groups including IT size in all patients had moderate to severe enlarged ITs.

Compared to preoperative scores, the PNIF, SNOT-22, and VAS scores at 2 weeks, 2 months, and 6 months were significantly better after undergoing MTR or ITP $(p<0.01)$ (Fig. 1). After 6 months of follow-up, there was a median increase of $57-58 \%$ in PNIF, a decrease in SNOT-22 scores by $53-80 \%$, and a decrease in 64-78\% VAS scores at the 6-month follow-up (Fig. 1). There was no difference in postoperative IT size in the MTR group compared to its preoperative size.

Postoperatively, PNIF in both groups improved when compared to the preoperative measurements $(p>0.05)$ (Fig. 1A). There was a greater percentage of improvement of $15-20 \%$ in SNOT-22 and VAS in patients treated with MTR compared to patients treated with ITP; however, this was not statistically significant ( $p>0.05)$ (Fig. 1B).

During the follow-up period, the PNIF measurements, SNOT22, and VAS scores between MTR and ITP groups did not show any significant difference $(p>0.05)$ (Fig. 1).

Based on the SNOT-22 and VAS, more patients in the MTR group $(83 \%, n=10)$ reported none or mild intermittent rhinitis symptoms, which did not require additional medications compared to the ITP group $(60 \%, n=6)$. However, this difference was not significant $(p>$ $0.05)$. The rest of the patients in both groups $(n=6)$ had persistent moderate to severe rhinitis requiring medications but reported to be satisfied with the outcome of surgery.

Operation time, pain scores, and length of stay in hospital did not show any statistical differences between the two surgical groups.

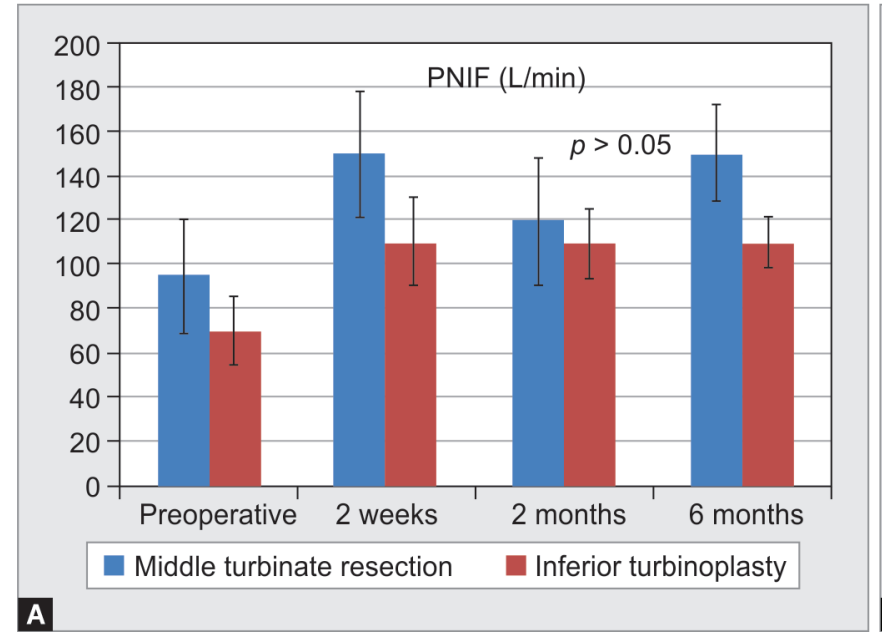

\section{Complications}

Postoperatively, there was significantly more bleeding in the ITP group compared to the MTR group based on the number of nasal bolster changes. Two patients (17\%) in the MTR group had more than one nasal bolster change overnight compared to six patients $(60 \%)$ in the ITP group $(p<0.05)$. However, there were none who required surgical intervention for bleeding.

There were two patients (17\%) in the MTR group who complained of mild hypernasality, which did not affect their lives. One patient (10\%) in the ITP group complained of intermittent epistaxis and mild crusting on the inferior turbinate that lasted for 3 weeks postoperatively. All symptoms of headache and ability to smell improved from moderate to severe initially to mild or none.

There were no episodes of atrophic rhinitis, adhesions, anosmia/ hyposmia, or failure requiring a revision procedure.

\section{Discussion}

\section{Comparing Outcomes of MTR and ITP}

This study has shown that even as early as the 2-week follow-up, both the MTR and ITP group had patients with PNIFs above $115 \mathrm{~L} /$ minute. The degree of improvement for PNIF was equivalent between both groups suggesting that the efficacy of MTR was comparable to ITP. This was further validated by the improvement in postoperative SNOT-22 and VAS scores in both groups. However, the higher percentage (15-20\%) of improvement of the SNOT-22 and VAS scores in the MTR group could be attributed to a slightly better preoperative score in MTR patients when compared to the ITP patients.

In the immediate postoperative period, the MTR group had $40 \%$ less postoperative bleeding compared to ITP group. This could be due to the MT being smaller than the IT thereby having a decreased amount of tissue loss and postoperative inflammation.

Studies have shown that the anterior MT is a key area for regulation of mucosal edema, nasal polyps, and vasoactive neuropeptides, such as, substance $P$, neurokinin $A$, and calcitonin gene-related peptide. ${ }^{24-27}$ Although smaller than the IT, White et al. found an increased in inflammatory protein receptors in the MT compared to IT in patients with chronic rhinosinusitis, which explains the increased potential for the MT to undergo polypoidal and edematous tissue changes. ${ }^{28}$ Therefore, the MT plays a

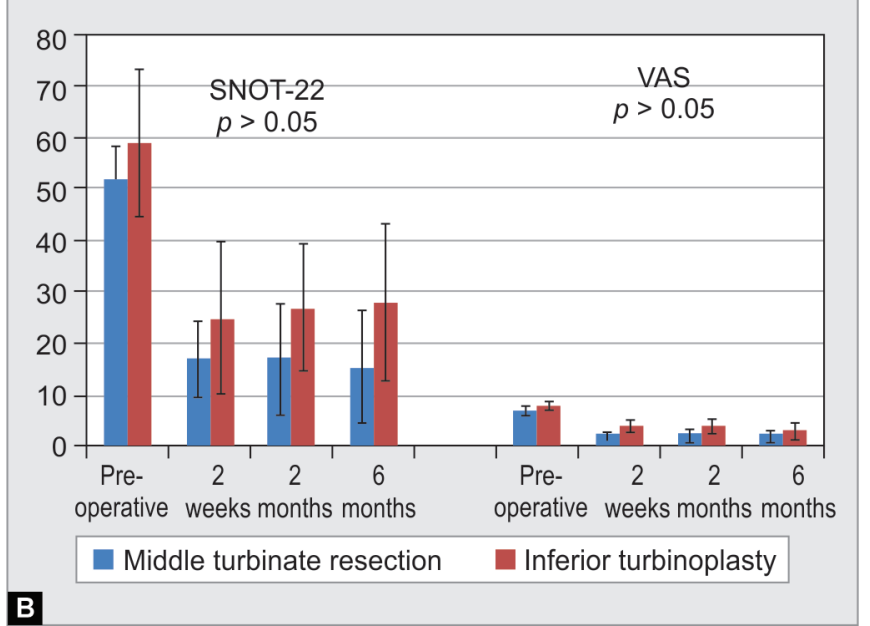

Figs $1 \mathrm{~A}$ and $\mathrm{B}$ : (A) Comparison of peak nasal inspiratory flow (PNIF) measurements between middle turbinate resection (MTR) and inferior turbinoplasty (ITP) groups; (B) Comparison of sinonasal outcome test-22 (SNOT-22) and visual analog scale (VAS) scores between MTR and ITP groups. Data given in median $\pm 95 \%$ confidence interval. Significance was only when groups were compared to preoperative scores. There was no significance between MTR and ITP groups 
significant role in nasal obstruction and that its resection could well be the reason that the MTR and ITP groups have equivalent postoperative PNIF results.

Marchioni et al. in 2008 prospectively performed complete MTRs in 22 patients with rhinosinusitis and found that the patients experienced less chances of recurrent disease. ${ }^{29}$ In our study, although there were significant improvements in sensory-related symptoms when comparing the pre- and postoperative outcomes, there was no statistical significant difference between removing the MT or IT. Postoperatively, both groups still had patients who needed to be dependent on oral antihistamines and intranasal corticosteroids.

There were no complains of anosmia/hyposmia, frontal headaches, or atrophic rhinitis encountered in this study, which was consistent with multiple studies that showed no side effects in removing the $\mathrm{MT}^{29,30}$

\section{Limitations of Surgical Intervention}

The major limitation of the present study was that it was a small, nonblinded trial without an independent assessor. However, we have been careful to ensure quality and consistency by having two clinicians reviewing each patient. We appreciate that this study is underpowered; however, it has given us enough information and confidence on the safety and efficacy of MTR to proceed with future studies in larger cohorts.

\section{Conclusion}

Middle turbinate resection has been shown in this preliminary pilot study to be as effective as ITP in the reduction of nasal obstructive and sensory-related symptoms of rhinitis and found to be a safe procedure in comparison to the traditional ITP.

\section{References}

1. Bonini $S$, Bonini $M$, Bousquet J, et al. Rhinitis and asthma in athletes: an ARIA document in collaboration with GA2LEN. Allergy. 2006;61(6):681-692. DOI: 10.1111/j.1398-9995.2006.01080.x.

2. Tan G, Ma Y, Li H, et al. Long-term results of bilateral endoscopic vidian neurectomy in the management of moderate to severe persistent allergic rhinitis. Arch Otolaryngol Head Neck Surg 2012;138(5):492497. DOI: 10.1001/archoto.2012.284.

3. Seidman MD, Gurgel RK, Lin SY, et al. Clinical practice guideline: Allergic rhinitis. Otolaryngol Head Neck Surg 2015;152(1 Suppl):S1S43. DOI: $10.1177 / 0194599814561600$.

4. Allergies in America. A telephone survey conducted in 2500 adults with allergic rhinitis. Healthstar Communications, Inc in partnership with Shulman, Ronca and Bucuvalas, Inc Allergies in America: A landmark survey on nasal allergy sufferers Executive summary Florham Park NJ: Altana Pharma US, Inc. 2006.

5. Barham HP, Thornton MA, Knisely A, et al. Long-term outcomes in medial flap inferior turbinoplasty are superior to submucosal electrocautery and submucosal powered turbinate reduction. Int Forum Allergy Rhinol 2015.

6. Prokopakis EP, Koudounarakis El, Velegrakis GA. Efficacy of inferior turbinoplasty with the use of $\mathrm{CO}(2)$ laser, radiofrequency, and electrocautery. Am J Rhinol Allergy 2014;28(3):269-272. DOI: 10.2500/ ajra.2014.28.4044.

7. Yankauer S. The Complete Sphenoethmoid Operation. The Laryngoscope. 1921;31(11):831-841. DOI: 10.1288/00005537192111000-00002.

8. Choby GW, Hobson CE, Lee S, et al. Clinical effects of middle turbinate resection after endoscopic sinus surgery: a systematic review. Am J Rhinol Allergy 2014;28(6):502-507. DOI: 10.2500/ajra.2014.28.4097.

9. Sozansky J, Houser SM. The physiological mechanism for sensing nasal airflow: a literature review. Int Forum Allergy Rhinol 2014;4(10):834-838. DOI: 10.1002/alr.21368.
10. Noruddin $\mathrm{NA}$, Saim $A B, C$ hua $K H$, et al. Human nasal turbinates as a viable source of respiratory epithelial cells using co-culture system versus dispase-dissociation technique. Laryngoscope 2007;117(12):2139-2145. DOI: 10.1097/MLG.0b013e3181453a1e.

11. Ruszymah BH, Izham BA, Heikal MY, et al. Human respiratory epithelial cells from nasal turbinate expressed stem cell genes even after serial passaging. Med J Malaysia 2011;66(5):440-442.

12. Lund VJ, Mackay IS. Staging in rhinosinusitus. Rhinology 1993;31(4):183-184.

13. Ottaviano G, Fokkens WJ. Measurements of nasal airflow and patency: a critical review with emphasis on the use of peak nasal inspiratory flow in daily practice. Allergy. 2016;71(2):162-174. DOI: 10.1111/all.12778.

14. Starling-Schwanz R, Peake HL, Salome CM, et al. Repeatability of peak nasal inspiratory flow measurements and utility for assessing the severity of rhinitis. Allergy 2005;60(6):795-800. DOI: 10.1111/j.13989995.2005.00779.x.

15. Ottaviano G, Lund VJ, Nardello E, et al. Comparison between unilateral PNIF and rhinomanometry in healthy and obstructed noses. Rhinology 2014;52(1):25-30. DOI: 10.4193/Rhin13.037.

16. Morgenstein KM, Krieger MK. Experiences in middle turbinectomy. Laryngoscope 1980;90(10 Pt 1):1596-1603. DOI: 10.1288/00005537198010000-00002.

17. Giacchi RJ, Lebowitz RA, Jacobs JB. Middle turbinate resection: issues and controversies. Am J Rhinol 2000;14(3):193-197. DOI 10.2500/105065800782102726.

18. Bousquet J, Khaltaev N, Cruz AA, et al. Allergic Rhinitis and its Impact on Asthma (ARIA) 2008 update (in collaboration with the World Health Organization, GA(2)LEN and AllerGen). Allergy 2008;63(Suppl 86):8-160. DOI: 10.1111/j.1398-9995.2007.01620.x.

19. Demoly P, Calderon MA, Casale T, et al. Assessment of disease control in allergic rhinitis. Clin Transl Allergy 2013;3(1):7.DOI: 10.1186/2045-7022-3-7.

20. Lange B, Thilsing T, Baelum J, et al. The Sinonasal Outcome Test 22 score in persons without chronic rhinosinusitis. Clin Otolaryngol. 2016;41(2):127-130. DOI: 10.1111/coa.12481.

21. Gillett S, Hopkins $C$, Slack R, et al. A pilot study of the SNOT 22 score in adults with no sinonasal disease. Clin Otolaryngol 2009;34(5): 467-469. DOI: 10.1111/j.1749-4486.2009.01975.x.

22. Bousquet PJ, Combescure C, Neukirch F, et al. Visual analog scales can assess the severity of rhinitis graded according to ARIA guidelines. Allergy 2007;62(4):367-372. DOI: 10.1111/j.1398-9995.2006.01276.x.

23. Farhood Z, Schlosser RJ, Pearse ME, et al. Twenty-two-item sino-nasal outcome test in a control population: a cross-sectional study and systematic review. Int Forum Allergy Rhinol 2015.

24. Havas TE, Lowinger DS. Comparison of functional endonasal sinus surgery with and without partial middle turbinate resection. Ann Otol Rhinol Laryngol 2000;109(7):634-640. DOI: 10.1177/000348940010900704.

25. Lundberg JM, Alving K, Lacroix JS, et al. Local and central reflex mechanisms in the neural control of airway microcirculation. Eur Respir J Suppl 1990;12:624s-628s.

26. Lacroix JS, Kurt AM, Pochon N, et al. Neutral endopeptidase activity and concentration of sensory neuropeptide in the human nasal mucosa. Eur Arch Otorhinolaryngol 1995;252(8):465-468. DOI: 10.1007/BF02114752.

27. Heppt W, Dinh QT, Cryer A, et al. Phenotypic alteration of neuropeptide-containing nerve fibres in seasonal intermittent allergic rhinitis. Clin Exp Allergy 2004;34(7):1105-1110. DOI: 10.1111/j.1365-2222.2004.01990.x.

28. White LC, Weinberger $P$, Coulson $\mathrm{H}$, et al. Why sinonasal disease spares the inferior turbinate: An immunohistochemical analysis. Laryngoscope 2015.

29. Marchioni D, Alicandri-Ciufelli M, Mattioli F, et al. Middle turbinate preservation versus middle turbinate resection in endoscopic surgical treatment of nasal polyposis. Acta Otolaryngol 2008;128(9):10191026. DOI: $10.1080 / 00016480701827541$

30. Soler ZM, Hwang PH, Mace J, et al. Outcomes after middle turbinate resection: revisiting a controversial topic. Laryngoscope 2010;120(4):832-837. DOI: 10.1002/lary.20812. 